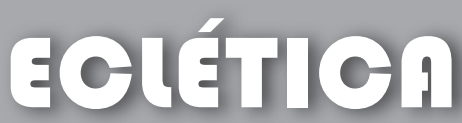 química
}

\section{DETERMINAÇÃO DE COEFICIENTE DE EXPANSÃO TÉRMICA DO BIODIESEL E SEUS IMPACTOS NO SISTEMA DE MEDIÇÃO VOLUMÉTRICO}

\author{
Douglas Queiroz Santos, Daniela Franco Vieira \\ Universidade Federal de Uberlândia - Avenida Engenheiro Diniz, 1178 - Caixa Postal: 593 - \\ CEP 38.400-902 - Uberlândia-Minas Gerais - Brasil \\ e-mail:douglasqueiroz@petrobras.com.br;danielafranco.tecline@petrobras.com.br
}

\begin{abstract}
Resumo: O objetivo deste trabalho foi determinar o coeficiente de expansão térmica do biodiesel a partir de dados experimentais de densidade em função da temperatura. Para tanto, foram utilizados alguns fundamentos da termodinâmica. O valor obtido para o coeficiente de expansão térmica após regressão linear para o biodiesel foi de $8,49 \times 10^{-4} \mathrm{C}^{-1}$, com um coeficiente de correlação igual a 0,9978 .
\end{abstract}

Palavras-chaves: Coeficiente de expansão térmica, dilatação térmica, biodiesel

\section{Introdução}

O aumento da demanda mundial por combustíveis líquidos, aquecimento global, segurança energética, vontade política por desenvolvimento nos campos agrícola, social e também energético são pontos que abrem novas áreas de interesse e oportunidades para pesquisas e desenvolvimento na Academia e na indústria, pois são as forças motoras responsáveis pelo renovado interesse na produção de biocombustíveis [1,2].

Com a inclusão do biodiesel na matriz energética do Brasil se faz necessário conhecer a magnitude do coeficiente de expansão, já que este é de extrema importância no calculo do faturamento do biodiesel nas indústrias e/ou bases de carregamento.

Cada material reage diferentemente a uma variação de temperatura [3]. Alguns materiais apresentam uma grande variação nas suas dimensões com o aumento da temperatura, enquanto outros praticamente não mudam suas dimensões [4], tal fato é explicado pelo coeficiente de expansão volumétrica [5].

Uma área promissora de estudos para caracterizar este bio-material é a medida das suas propriedades térmicas. Estas propriedades revelam informações importantes sobre o comportamento físico-químico no material e certamente pode ser um caminho adicional nas metodologias de caracterização de biodiesel [6].

O coeficiente de expansão térmica $(\beta)$ também chamado de coeficiente de expansão volumétrica ou expansividade volumétrica indica a variação de volume $(\mathrm{V})$ provocada pela variação da temperatura (T) enquanto a pressão (P) permanece constante [4,7]:

$$
\beta=\frac{1}{V} \times \frac{\partial V}{\partial T}
$$

O coeficiente de expansão térmica $(\beta)$ também chamado de compressibilidade isobárica $[8,9]$, uma vez que a pressão é constante. Entre o coeficiente de expansão térmica e a energia de ligação química entre os átomos existe uma boa correlação, onde materiais em que as ligações químicas são fortes apresentam o coeficiente de expansão térmica baixo. Isto porque a dilatação térmica está associada à variação assimétrica da energia (ou força) de ligação com a distância entre os 
átomos. Ou seja, durante o aquecimento os átomos do material aumentam a freqüência e a amplitude de vibração e como as forças de repulsão são sempre maiores que as de atração, a distância média entre os átomos também aumenta [7].

\section{Material e Métodos}

Durante os meses de abril, maio, junho e julho do ano de 2009 coletaram-se amostras de biodiesel das seguintes Empresas Agrosoja, Fiagril, Caibiense, Bracol, Binatural, Caramuru, Granol, Biopar e Fertibom. Com essas empresas de acordo com a ANP, temos biodiesel de rota etilíca e metílica com origem vegetal e animal, ou seja, temos uma grande variabilidade nas origens.

A tabela 1 indica os dados experimentais de densidade do biodiesel em função da temperatura, nestes dados foi aplicado os testes de Chauvenet, Dixox e Grubbs para verificação de outliers. Após este procedimento, os dados foram aplicados à equação (viii) onde foi possível determinar $o$ coeficiente de expansão térmica a partir da análise de regressão linear, sendo o coeficiente angular da reta obtida pelo gráfico de $\ln (\mathrm{d} 0 / \mathrm{d})$ versus (T-T0) corresponde numericamente ao valor do coeficiente de expansão térmica para o biodiesel. Nos cálculos, consideraram-se como $\mathrm{T} 0$ as temperaturas de $10,0^{\circ} \mathrm{C}$ e $\mathrm{d} 0$, as densidades dos biodiesel nesta temperatura.

Tabela 1. Efeito da temperatura sobre a densidade do biodiesel.

\begin{tabular}{|c|c|c|c|c|c|c|c|c|c|c|}
\hline Temperatura $\left({ }^{\circ} \mathrm{C}\right)$ & 10,0 & 15,0 & 20,0 & 25,0 & 30,0 & 35,0 & 40,0 & 45,0 & 50,0 & Amostra \\
\hline \multirow[t]{31}{*}{ Densidade $\left(\mathrm{Kg} \times \mathrm{m}^{3}\right)$} & 890,0 & 886,1 & 882,1 & 878,5 & 875,1 & 871,4 & 867,9 & 864,2 & 860,7 & 1 \\
\hline & 889,9 & 886,2 & 882,1 & 878,2 & 874,9 & 871,3 & 867,7 & 864,1 & 860,4 & 2 \\
\hline & 890,0 & 886,4 & 882,2 & 878,7 & 875,0 & 871,5 & 867,9 & 864,2 & 860,6 & 3 \\
\hline & 890,3 & 886,5 & 882,7 & 878,9 & 875,3 & 871,7 & 868,1 & 864,5 & 860,9 & 4 \\
\hline & 890,5 & 886,6 & 882,1 & 878,8 & 875,2 & 871,6 & 868,0 & 864,4 & 860,7 & 5 \\
\hline & 890,5 & 886,3 & 882,6 & 878,9 & 875,3 & 871,5 & 868,0 & 864,4 & 860,7 & 6 \\
\hline & 890,1 & 886,4 & 882,7 & 879,2 & 874,8 & 871,2 & 867,6 & 863,9 & 860,3 & 7 \\
\hline & 889,2 & 885,3 & 881,7 & 878,7 & 874,3 & 870,6 & 867,0 & 863,4 & 859,7 & 8 \\
\hline & 890,6 & 886,6 & 882,6 & 878,9 & 875,4 & 871,7 & 868,1 & 864,4 & 860,8 & 9 \\
\hline & 890,7 & 887,0 & 882,7 & 879,1 & 875,3 & 871,7 & 868,1 & 864,5 & 860,9 & 10 \\
\hline & 890,3 & 886,3 & 882,4 & 879,5 & 875,3 & 871,5 & 867,9 & 865,5 & 860,7 & 11 \\
\hline & 889,1 & 885,4 & 881,7 & 878,3 & 874,4 & 870,8 & 867,2 & 863,6 & 860,0 & 12 \\
\hline & 890,0 & 886,3 & 882,4 & 878,7 & 875,1 & 871,5 & 867,8 & 864,2 & 860,6 & 13 \\
\hline & 889,8 & 886,1 & 882,3 & 878,7 & 875,0 & 871,4 & 867,3 & 864,2 & 860,6 & 14 \\
\hline & 889,8 & 885,9 & 882,1 & 878,5 & 874,9 & 871,2 & 867,6 & 864,0 & 860,4 & 15 \\
\hline & 890,1 & 885,8 & 882,0 & 878,4 & 874,8 & 871,2 & 867,5 & 863,9 & 860,3 & 16 \\
\hline & 889,5 & 885,5 & 881,8 & 878,1 & 874,5 & 870,9 & 867,3 & 863,7 & 860,0 & 17 \\
\hline & 889,5 & 885,4 & 881,4 & 877,8 & 874,2 & 870,5 & 867,0 & 863,3 & 859,7 & 18 \\
\hline & 889,3 & 885,3 & 881,7 & 878,6 & 874,3 & 870,7 & 867,0 & 863,4 & 859,8 & 19 \\
\hline & 889,1 & 885,4 & 881,7 & 878,0 & 874,5 & 870,8 & 867,2 & 863,6 & 860,3 & 20 \\
\hline & 889,4 & 885,6 & 881,6 & 878,0 & 874,4 & 870,7 & 867,1 & 863,4 & 859,8 & 21 \\
\hline & 889,2 & 885,1 & 881,5 & 877,8 & 874,2 & 870,5 & 866,9 & 863,2 & 859,6 & 22 \\
\hline & 889,8 & 886,2 & 881,8 & 878,1 & 874,4 & 871,1 & 867,2 & 863,5 & 860,0 & 23 \\
\hline & 889,7 & 886,2 & 881,8 & 878,1 & 874,5 & 870,8 & 867,3 & 863,6 & 860,0 & 24 \\
\hline & 889,7 & 886,0 & 881,7 & 878,0 & 874,4 & 870,7 & 867,1 & 863,5 & 859,9 & 25 \\
\hline & 889,7 & 886,0 & 881,6 & 878,0 & 874,4 & 870,7 & 867,1 & 863,5 & 859,9 & 26 \\
\hline & 889,6 & 885,8 & 881,6 & 878,0 & 874,4 & 870,7 & 867,1 & 864,2 & 859,8 & 27 \\
\hline & 889,5 & 885,6 & 881,5 & 877,8 & 874,2 & 870,6 & 867,0 & 863,3 & 859,7 & 28 \\
\hline & 889,8 & 885,8 & 881,7 & 878,1 & 874,5 & 870,8 & 867,2 & 863,6 & 860,0 & 29 \\
\hline & 889,6 & 886,0 & 881,7 & 878,0 & 874,4 & 870,8 & 867,2 & 863,5 & 859,9 & 30 \\
\hline & 889,7 & 885,9 & 881,7 & 878,1 & 874,5 & 870,8 & 867,3 & 863,5 & 860,6 & 31 \\
\hline
\end{tabular}


continuação da Tabela 1. Efeito da temperatura sobre a densidade do biodiesel.

\begin{tabular}{|c|c|c|c|c|c|c|c|c|c|c|}
\hline Temperatura $\left({ }^{\circ} \mathrm{C}\right)$ & 10,0 & 15,0 & 20,0 & 25,0 & 30,0 & 35,0 & 40,0 & 45,0 & 50,0 & Amostra \\
\hline & 889,6 & 885,9 & 881,9 & 878,1 & 874,5 & 870,8 & 867,2 & 863,5 & 859,9 & 32 \\
\hline & 889,1 & 885,4 & 881,7 & 878,1 & 874,5 & 870,8 & 867,3 & 863,6 & 860,0 & 33 \\
\hline & 889,8 & 885,9 & 881,7 & 877,9 & 874,3 & 870,7 & 867,1 & 863,5 & 859,9 & 34 \\
\hline & 889,8 & 885,9 & 881,7 & 878,1 & 874,5 & 870,8 & 867,2 & 863,6 & 860,0 & 35 \\
\hline & 889,5 & 885,6 & 881,4 & 877,7 & 874,0 & 870,4 & 866,8 & 863,1 & 859,6 & 36 \\
\hline & 889,1 & 885,3 & 881,6 & 878,0 & 874,3 & 870,7 & 867,1 & 863,4 & 859,8 & 37 \\
\hline & 889,0 & 885,3 & 881,6 & 877,9 & 874,3 & 870,7 & 867,1 & 863,4 & 859,8 & 38 \\
\hline & 889,2 & 885,3 & 881,6 & 878,7 & 874,4 & 870,8 & 867,1 & 863,5 & 859,9 & 39 \\
\hline & 889,0 & 885,1 & 881,2 & 877,5 & 873,9 & 870,2 & 866,6 & 863,0 & 859,3 & 40 \\
\hline & 888,7 & 884,7 & 880,8 & 877,1 & 873,5 & 869,9 & 866,3 & 862,7 & 859,0 & 41 \\
\hline & 889,2 & 885,5 & 881,8 & 878,1 & 874,5 & 870,8 & 867,3 & 863,7 & 860,0 & 42 \\
\hline & 889,1 & 885,4 & 881,5 & 877,8 & 874,2 & 870,5 & 867,0 & 863,4 & 859,7 & 43 \\
\hline & 888,2 & 884,6 & 880,9 & 877,3 & 873,6 & 870,0 & 866,4 & 862,8 & 859,2 & 44 \\
\hline & 888,6 & 885,3 & 881,3 & 877,7 & 874,1 & 870,4 & 866,8 & 863,2 & 859,6 & 45 \\
\hline & 888,9 & 885,5 & 881,6 & 878,0 & 874,3 & 870,7 & 867,1 & 863,5 & 859,8 & 46 \\
\hline & 889,1 & 885,5 & 881,7 & 878,1 & 874,4 & 870,8 & 867,2 & 863,6 & 860,0 & 47 \\
\hline & 889,0 & 885,5 & 881,7 & 878,1 & 874,4 & 870,8 & 867,3 & 863,6 & 860,0 & 48 \\
\hline & 889,1 & 885,3 & 881,8 & 878,1 & 874,5 & 870,9 & 867,3 & 863,7 & 860,1 & 49 \\
\hline & 888,9 & 885,3 & 881,7 & 878,0 & 874,4 & 870,8 & 867,2 & 863,5 & 860,0 & 50 \\
\hline & 889,1 & 885,5 & 881,8 & 877,9 & 874,5 & 870,9 & 867,3 & 863,6 & 860,0 & 51 \\
\hline & 889,0 & 885,4 & 881,7 & 878,0 & 874,3 & 870,7 & 867,1 & 863,5 & 859,8 & 52 \\
\hline & 888,5 & 884,8 & 881,2 & 877,4 & 873,8 & 870,1 & 866,6 & 862,9 & 859,3 & 53 \\
\hline & 889,2 & 885,8 & 881,7 & 878,1 & 874,5 & 870,8 & 867,2 & 863,6 & 860,0 & 54 \\
\hline & 888,7 & 885,2 & 881,4 & 877,8 & 874,1 & 870,5 & 866,9 & 863,2 & 859,7 & 55 \\
\hline & 888,9 & 885,3 & 881,6 & 877,8 & 874,4 & 870,7 & 867,1 & 863,5 & 859,9 & 56 \\
\hline & 888,6 & 884,9 & 881,3 & 877,7 & 874,1 & 870,5 & 866,8 & 863,2 & 859,6 & 57 \\
\hline & 888,9 & 885,2 & 881,7 & 878,1 & 874,4 & 870,8 & 867,1 & 863,5 & 859,9 & 58 \\
\hline & 889,0 & 885,4 & 881,8 & 878,1 & 874,5 & 870,8 & 867,2 & 863,6 & 860,0 & 59 \\
\hline & 889,1 & 885,4 & 881,7 & 878,0 & 874,4 & 870,7 & 867,1 & 863,5 & 859,9 & 60 \\
\hline & 889,1 & 885,6 & 881,8 & 878,1 & 874,5 & 870,8 & 867,3 & 863,6 & 860,0 & 61 \\
\hline & 888,1 & 884,5 & 880,9 & 877,1 & 873,5 & 869,9 & 866,3 & 862,6 & 859,0 & 62 \\
\hline & 889,0 & 885,4 & 881,7 & 878,1 & 874,0 & 870,8 & 867,2 & 863,6 & 860,0 & 63 \\
\hline & 889,3 & 885,3 & 881,7 & 878,1 & 874,5 & 870,8 & 867,2 & 863,6 & 860,0 & 64 \\
\hline & 888,9 & 885,7 & 881,6 & 878,0 & 874,3 & 870,7 & 867,0 & 863,5 & 859,9 & 65 \\
\hline & 889,1 & 885,5 & 881,8 & 878,1 & 874,5 & 870,9 & 867,3 & 863,7 & 860,1 & 66 \\
\hline & 883,7 & 879,7 & 875,9 & 873,2 & 868,8 & 865,1 & 861,5 & 857,8 & 854,2 & 67 \\
\hline & 889,3 & 885,5 & 881,3 & 877,7 & 874,0 & 870,4 & 866,8 & 863,2 & 859,6 & 68 \\
\hline & 889,2 & 885,3 & 881,3 & 877,6 & 874,0 & 870,4 & 866,7 & 863,1 & 859,5 & 69 \\
\hline & 888,6 & 884,7 & 880,6 & 877,0 & 873,3 & 869,7 & 866,1 & 862,4 & 858,9 & 70 \\
\hline & 890,0 & 886,0 & 881,9 & 878,3 & 874,6 & 871,0 & 867,4 & 863,8 & 860,1 & 71 \\
\hline & 883,5 & 879,3 & 875,6 & 871,9 & 868,3 & 864,7 & 861,0 & 857,4 & 853,8 & 72 \\
\hline & 884,4 & 879,6 & 876,1 & 872,4 & 868,9 & 865,2 & 861,7 & 858,0 & 854,3 & 73 \\
\hline & 883,7 & 879,1 & 875,4 & 871,7 & 868,1 & 864,4 & 860,9 & 857,2 & 853,5 & 74 \\
\hline & 884,5 & 879,8 & 876,2 & 872,5 & 868,9 & 865,3 & 861,7 & 858,1 & 854,4 & 75 \\
\hline & 888,1 & 884,6 & 880,9 & 877,3 & 873,7 & 870,0 & 866,4 & 862,8 & 859,1 & 76 \\
\hline & 888,1 & 884,5 & 880,7 & 877,0 & 873,5 & 869,8 & 866,3 & 862,5 & 859,0 & 77 \\
\hline & 888,2 & 884,7 & 880,9 & 877,3 & 873,7 & 870,1 & 866,5 & 862,7 & 859,2 & 78 \\
\hline & 888,5 & 884,6 & 880,9 & 877,2 & 873,6 & 870,1 & 866,4 & 862,7 & 859,2 & 79 \\
\hline & 887,8 & 885,0 & 880,6 & 876,9 & 873,3 & 869,6 & 866,0 & 862,4 & 858,8 & 80 \\
\hline
\end{tabular}




\section{Resultados e Discussão}

Conforme figura 1 a análise de regressão linear forneceu como resultado para o coeficiente de expansão térmica $(\beta)$ do biodiesel o valor de $8,49 \times 10^{-4}$ ${ }^{\circ} \mathrm{C}^{-1}$, com um coeficiente de correlação igual a 0,9978 .

O coeficiente de correlação tem por objetivo avaliar a "qualidade" do ajuste. Assim para um coeficiente de correlação mais próximo a 1, melhor a "qualidade" do ajuste da função aos pontos do diagrama de dispersão.

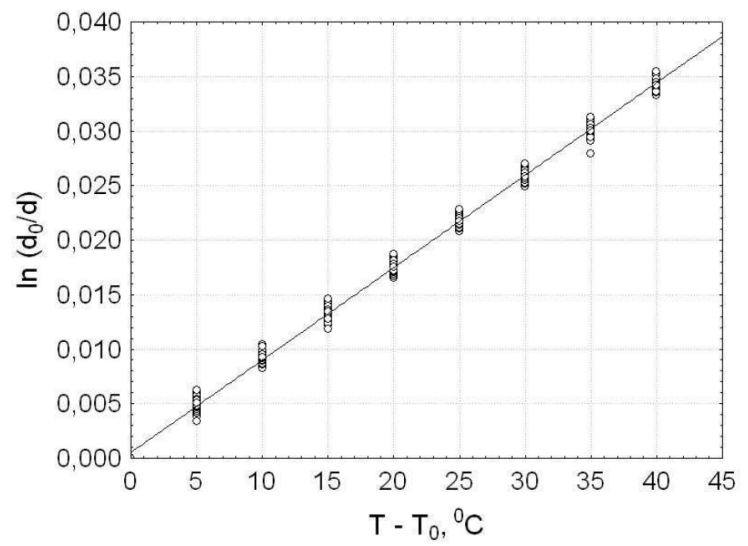

Figura 1. Gráfico de $\ln \left(\mathrm{d}_{0} / \mathrm{d}\right)$ versus $\left(T-\mathrm{T}_{0}\right)$ para biodiesel.

Observa-se na figura 2 que não existe padrão de comportamento, podendo-se concluir que a variância dos erros é constante.

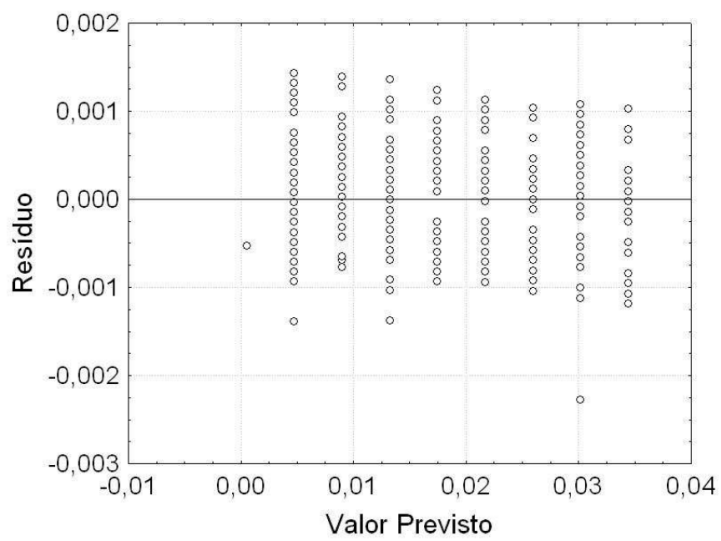

Figura 2. Resíduo versus valor previsto para os dados da tabela 1 .
Pela figura 3 podemos observar que os erros de ajustamento estão independentes e normalmente distribuídos em torno da reta. Quanto mais próximos os pontos experimentais estiverem da linha contínua, mais válida a suposição de normalidade dos resíduos.

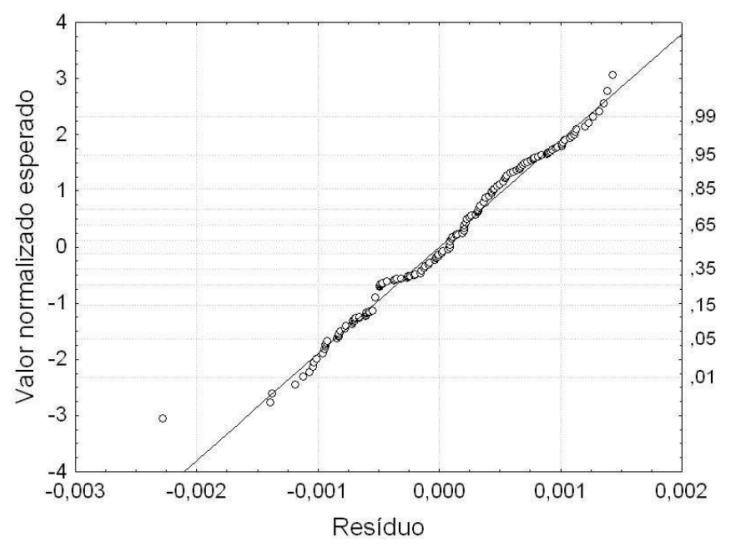

Figura 3. Distribuição dos resíduos em torno da reta que indica normalidade.

\section{Conclusões}

A utilização de dados experimentais de densidade em função da temperatura associada a alguns conceitos da termodinâmica permitiu predizer o coeficiente de expansão térmica do biodiesel, parâmetro este de extrema importância para estimar outras propriedades físico-químicas entre elas a entalpia de vaporização $[5,10]$. Considerando que o coeficiente de expansão térmica do biodiesel é $8,49 \times 10^{-4}{ }^{\circ} \mathrm{C}^{-1}$, para um aumento de temperatura de $10,0^{\circ} \mathrm{C}$, o aumento no volume do biodiesel é da ordem de 8,5 litros para cada mil litros.

Sendo assim o volume ocupado pelo biodiesel no interior de tanques sofre forte influência da temperatura e para viabilizar o comércio destes produtos e valorizar todas as operações comerciais e estabelecer os preços com os volumes referidos à temperatura de $20,0^{\circ} \mathrm{C}$, temperatura de referência no Brasil, se faz necessário corrigir o volume à temperatura ambiente para a temperatura de referência, sendo necessário para isso o coeficiente de expansão térmica neste trabalho. 
Douglas Queiroz Santos, Daniela Franco Vieira. Determination of coefficient of thermal expansion of biodiesel and their impacts on system volumetric measurement.

Abstract: The purpose of this study was to determine the coefficient of thermal expansion of the biodiesel from the experimental data of density as a function of temperature. For this, we used some fundamentals of thermodynamics. The value obtained for the coefficient of thermal expansion after the linear regression for biodiesel was $8.49 \times 10^{-4} \circ \mathrm{C}^{-1}$, with a correlation coefficient equal to 0.9978 .

Keywords: Coefficient of thermal expansion, thermal expansion, biodiesel.

\section{Referências}

[1] I. P. Lôbo, S. L. C. Ferreira, R. S. Cruz, Química Nova 32(6) (2009) 1596-1608.

[2] M. J. Dabdoub, J. L. Bronzel, M. A. Rampin, Química Nova 32(3) (2009) 776-792.

[3] F. Cabral, A. Lago, Fícica 2. Harbra, São Paulo, 2002, p.17-21.

[4] W. D. Callister, Ciência e Engenharia de Materiais: uma introdução, Livros Técnicos e Científicos Editora, Rio de Janeiro, 2002, p.450.

[5] E. C. Elizondo, G. Lutz, J. F. M. Segreda, Jornal of Phisical Organic Chemistry 19(1) (2006) 744-747.

[6] L. M. R. Antón, C. Aparicio, B. Guignon, P. D. Sanz,
ScienceDirect 87(1) (2008) 1934-1940.

[7] A. F. Padilha, Materiais de Engenharia: microestrutura e propriedades, Hemus, São Paulo, 1997,

p. 289-301.

[8] F. W. Schimidt, R. E. Henderson, C. H. Wolgemuth, Introdução às Ciências Térmicas: Termodinâmica, Mecânica dos Fluidos e Transferência de Calor, Edgard Blücher, São Paulo, 2001, p. 50-51.

[9] J. M. Smith, H. C. V. Ness, Introdução à Termodinâmica da Engenharia Química, Guanabara Dois, Rio de Janeiro, 1985, p. 53-57.

[10] R. E. Sonntag, C. Borgnakke, G. J. V. Wylen, Fundamentos da Termodinâmica, Edgard Blücher, São Paulo, 1998, p.57. 
